FERREIRA, L.FL, BRACCINI, P. e FRANKLIN, N. Escala de dor em pequenos animais - revisão de literatura. PUBVET, Londrina, V. 8, N. 1, Ed. 250, Art. 1651, Janeiro, 2014.

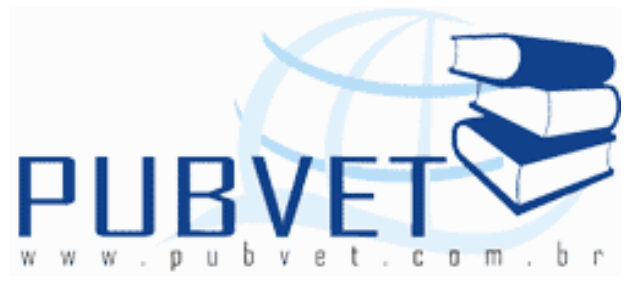

PUBVET, Publicações em Medicina Veterinária e Zootecnia.

\title{
Escala de dor em pequenos animais - revisão de literatura
}

Luiz Fernando Lucas Ferreira ${ }^{1}$, Paula Braccini ${ }^{2}$ e Natália Franklin ${ }^{2}$

${ }^{1}$ Aluno do Programa de Pós-Graduação em Cirurgia Veterinária da FCAV UNESP - Jaboticabal.

${ }^{2}$ Alunas do curso de graduação em Medicina Veterinária da PUC - Minas Betim

\section{Resumo}

Estudaram-se as principias escalas para dor em pequenos animais descritas pela literatura. Observou-se o consenso da necessidade de treinamento e experiência clínica dos avaliadores. A utilização de modelos que combinam a avaliação de parâmetros fisiológicos, objetivos, com alterações comportamentais, subjetivos, sinaliza para a melhor forma de entender e avaliar um paciente com dor.

Palavras-chave: escala, dor, pequenos animais

\section{Pain evaluation in small animals - review}

\section{Abstract}

We studied all of the major scales for pain in small animals described in the literature. Observed the consensus of the need for training and clinical experience of the evaluators. The use of models that combine the assessment 
FERREIRA, L.FL, BRACCINI, P. e FRANKLIN, N. Escala de dor em pequenos animais - revisão de literatura. PUBVET, Londrina, V. 8, N. 1, Ed. 250, Art. 1651, Janeiro, 2014.

of physiological parameters with behavioral parameters, points to the best way to understand and assess a patient with pain.

Keywords: pain, scale, small animal

\section{INTRODUÇÃO}

A dor sempre foi motivo de estudo, tanto na medicina humana como na medicina veterinária. Contudo, este tema nunca foi tão investigado como nos dias atuais. A procura de uma escala para dor, ou uma forma de mensura-la tem sido o objetivo de muitas pesquisas. Em seres humanos, escalas numéricas ou questionários já estão disponíveis, porém, quando se trata do paciente pediátrico e de animas, avaliar a dor ou tentar mensura-la, ainda é muito difícil, pois é realizada subjetivamente.

O conceito de bem estar animal, seja para pesquisas ou em tratamentos clínicos e cirúrgicos, vem conscientizando o médico veterinário a não negligenciar a dor, bem como não utilizar de terapêutica excessiva para tratamento da mesma.

A possibilidade de avaliar alterações fisiológicas, objetivas, e comportamentais, ou subjetivas, através de um modelo matemático, pode ser um indicador na avaliaçãoda dor nos animais de companhia.

Sabe-se pela literatura que estímulos álgicos irão produzir alteração na frequência cardíaca (FC), na temperatura corporal (TC) e na frequência respiratória (FR). Estes parâmetros fisiológicos podem ser mensurados, objetivamente, e são importantes para o processo de avaliação da dor. Em contra partida, os parâmetros comportamentais como agressividade, deambulação, micção, defecação, ingestão de água e ração são passíveis de serem avaliados somente por meios subjetivos (HEYLLER et al., 2007).

Este estudo tem por objetivo revisar as várias escalas de dor para animais de companhia descritas na literatura. 
FERREIRA, L.FL, BRACCINI, P. e FRANKLIN, N. Escala de dor em pequenos animais - revisão de literatura. PUBVET, Londrina, V. 8, N. 1, Ed. 250, Art. 1651, Janeiro, 2014.

\section{REVISÃO DE LITERATURA}

A avaliação da dor em animais é uma tarefa desafiadora para a medicina veterinária (CASTRO, 2008). Este procedimento em animais, bem como em neonatos humanos, não é simples, pela dificuldade de interpretar o comportamento dos mesmos, uma vez que não existe comunicação verbal (LUNA, 2008). Os animais com dor podem ficar deprimidos e indiferentes ao meio, ou ao contrário, extremamente agressivos e agitados. Pode-se observar também dificuldade para repousar, inapetência, tentativas de lamber, morder e / ou coçar a região dolorida. Há alterações na postura corporal com relutância em deitar-se e movimentar-se, para proteger a área dolorida (IMAGAWA, 2006). Uma avaliação completa da dor deve abranger uma observação não interativa, empreendida à distância; e uma apreciação interativa envolvendo um estímulo que provoque uma resposta do paciente. Para que a avaliação da dor seja mais fidedigna possível, ela deve ser seriada e realizada, de preferência, por um mesmo observador (CÔRTES, 2006).

Segundo o guia de 2007, publicado pelaAAHA/AAFP (American Animal Hospital Association /American AssociationofFelinePractioners), os sinais gerais de dor são: alteração de comportamento, manifestação anormal de comportamento, reação ao toque e alteração de parâmetros fisiológicos. Respectivamente, podem ser exemplificados como letargia, relutância em andar e falta de apetite. Vocalização, agressividade e alteração de postura. Aumento da tensão muscular ao toque e ou palpação da área incisionada (sitio cirúrgico) ou mesmo da área próxima. Aumento da frequência cardíaca, frequência respiratória, temperatura corporal e dilatação da pupila (HELLYER et al., 2007).

Para CONZENIUS et al. (1997), os parâmetros objetivos como a frequência cardíaca, frequência respiratória e temperatura não são confiáveis para avaliar a dor, bem como a mensuração de fatores humorais como epinefrina, norepinefrina e cortisol. 
FERREIRA, L.FL, BRACCINI, P. e FRANKLIN, N. Escala de dor em pequenos animais - revisão de literatura. PUBVET, Londrina, V. 8, N. 1, Ed. 250, Art. 1651, Janeiro, 2014.

Várias escalas têm sido introduzidas na prática clínica de pequenos animais (LUNA, 2008), caracterizadas como métodos subjetivos de quantificação da dor (IMAGAWA, 2006). Estas escalas se baseiam principalmente no comportamento do paciente e as mais utilizadas para a quantificação da dor em animais são:

Escala Analógica Visual (VAS)

Escala Numérica Visual (RNS)

Escala Simples Descritiva (SDS) (MATHEWS, 2000).

A VAS é um método que possui um formato de linha reta não numerada, apresentando o comprimento de $10 \mathrm{~cm}$, sendo o início da linha representado pelo 0 (zero), interpretado "ausência de dor" e a outra extremidade é representada pelo 10 (dez), indicando "pior ou maior dor" experimentada pelo paciente. O observador treinado deve colocar um ponto nesta reta durante a avaliação, supondo o grau de dor que o animal esteja padecendo (CÔRTES, 2006; CASTRO, 2008).

A RNS é um método similar de quantificação da dor, todavia o avaliador não marca um ponto em uma reta, e sim, promove uma pontuação numérica de acordo com as atividades do paciente, que são divididas dentro de categorias de comportamentos (CASTRO, 2008).

A SDS consiste em um método de avaliação da dor de menor complexidade, podendo ser dividida em quatro ou mais categorias: ausência de dor, dor leve, dor moderada e dor grave (CASTRO, 2008).

A escala utilizada na faculdade de Medicina Veterinária da Universidade de Guelph, Ontário, proposta por MATHEWS (2000), possui uma graduação de 0 a 10 e baseia-se na avaliação do comportamento do animal. Medidas objetivas para a avaliação da dor podem ser utilizadas, a fim de mensurar os parâmetros fisiológicos como: freqüência cardíaca e respiratória, pressão arterial e temperatura (IMAGAWA, 2006). Já as avaliações minuciosas de fatores endógenos envolvem determinações bioquímicas, como, por exemplo, 
FERREIRA, L.FL, BRACCINI, P. e FRANKLIN, N. Escala de dor em pequenos animais - revisão de literatura. PUBVET, Londrina, V. 8, N. 1, Ed. 250, Art. 1651, Janeiro, 2014.

a dosagem de cortisol, que pode auxiliar no diagnóstico da dor em animais (CASTRO, 2008).

Outras escalas que avaliam a analgesia e a classificam numericamente, para a formação de um escore, também podem ser empregadas, como a escala de análise descritiva, proposta por Lascelleset al. (1994), que utiliza, além da observação do comportamento, a palpação como método de diagnóstico. A escala, descrita por Firth e Haldene (1999), leva em consideração as medidas dos parâmetros fisiológicos e comportamentais associados a uma mesma escala.

Escala de dor da Universidade de Melbourne (UMPS): É uma escala baseada em respostas comportamentais e fisiológicas específicas. Inclui múltiplas descrições organizadas em seis categorias de parâmetros ou comportamentos relacionados com a dor. A UMPS apresenta uma maior precisão relativamente à VAS, à SDS, à NRS e permite diferenciar a importância de certos comportamentos ou parâmetros. A avaliação de múltiplos fatores aumenta a sua sensibilidade e especificidade. No entanto, pode não ser suficientemente sensível para detectar pequenas mudanças de comportamento, principalmente quando a avaliação é feita periodicamente. Esta escala foi desenhada especificamente para a avaliação pós-cirúrgica em cães, podendo, por isso, não se adequar a outro tipo de situações e espécies. Outra desvantagem é o fato de não considerar reações secundárias à anestesia (p.e. disforia e sedação persistente). Exige também que o observador conheça o estado mental normal do animal antes da cirurgia e anestesia (HANSEN, 2003; HELLYER, 2005).

Escala Composta de Dor de Glasgow (GCMPS): baseia-se em sinais comportamentais considerados como relacionados com a dor no cão. Os comportamentos incluídos nesta escala são o resultado de um questionário respondido por veterinários, em que os termos utilizados para a descrição da dor foram filtrados e reduzidos a palavras específicas e expressões, sendo validados por métodos estatísticos e testadas quanto à sua validade, 
FERREIRA, L.FL, BRACCINI, P. e FRANKLIN, N. Escala de dor em pequenos animais - revisão de literatura. PUBVET, Londrina, V. 8, N. 1, Ed. 250, Art. 1651, Janeiro, 2014.

sensibilidade e confiabilidade(MURRELL, et al., 2008). Nesta escala, a interpretação e a variabilidade, por parte do observador, está mais limitada, promovendo um aumento da sua precisão, isto é, o observador é questionado no sentido de verificar se determinado comportamento se encontra ou não presente no momento da avaliação do paciente. Não são incluídos dados fisiológicos, tornando a sua utilização mais simples. Por outro lado, esta escala carece de um sistema de pontuação que permita de forma simples, acompanhar a evolução do paciente (HELLYER, 2005).

Escala Composta de Dor de Glasgow - Forma Abreviada (GCMPS-SF): é o resultado de uma modificação da GCMPS, realizada no sentido de ser mais facilmente aplicada num ambiente clínico, e ser uma ferramenta auxiliar na tomada de decisões relacionadas aos cães com dor aguda. Tem como principal vantagem relativamente à GCMPS,a inclusão de um sistema de pontuação (MICH \& HELLYER, 2009; MORTON, et al., 2005).

Escala de Dor da Universidade de Colorado: Deriva da UMPS, GCMPS, GCMPS-SF e SDS. O seu formato incorpora os parâmetros mais práticos de cada uma das escalas, apresentando-se numa única folha e com um grafismo apelativo. A escala utiliza um período de observação e a sua abordagem é divida em 2 fases. Na primeira, o paciente é avaliado em descanso, na sua jaula, e a uma distância que não perturbe o animal. Seguidamente, é avaliado o estado geral do paciente, a sua resposta à palpação da área cirúrgica e tensão corporal. Associa ainda uma escala numérica de 0-4, divida em quartos, e, por vezes, imagens realísticas de algumas atitudes corporais possíveis do animal em dor. As vantagens desta escala relacionam-se com sua fácil utilização e possibilidade de utilização em cães e gatos, e, a adição da avaliação da tensão corporal, sendo a interpretação subjetiva por parte do observador reduzida. Faltam, no entanto, estudos clínicos que permitam a sua validação (MICH \& HELLYER, 2009). 
FERREIRA, L.FL, BRACCINI, P. e FRANKLIN, N. Escala de dor em pequenos animais - revisão de literatura. PUBVET, Londrina, V. 8, N. 1, Ed. 250, Art. 1651, Janeiro, 2014.

\section{CONSIDERAÇÕES FINAIS}

A possibilidade de mensurar a dor em animais de estimação ainda é um desafio par a medicina veterinária. Parâmetros objetivos, fisiológicos, associados a observação do comportamento animal, sinalizam para um conceito comum da melhor forma de avaliação da dor em animais de companhia. O treinamento dos avaliadores e experiência clínica profissional são quesitos relevantes na validação de um processo de escala de dor.

\section{REFERÊNCIAS BIBLIOGRÁFICAS}

CASTRO, D.S. Comparação entre o efeito analgésico da morfina e do tramadolepidural em gatos (feliscatusdomesticus). Rio de Janeiro, 2008. 48f. Dissertação. (Mestrado em Ciências Veterinárias). Universidade Federal Rural do Rio de Janeiro,Rio de Janeiro, 2008.

CONZEMIUS MG, HILL CM, SAMMARCO JL, PERKOWSKI SZ. Correlation between subjective and objective measures used to determine severity of postoperative pain in dogs.. Journal of the American Veterinary Medical Association, v.210, n. 11, p. 1619-1622, 1997.

CÔRTES, L.A. Avaliação do perfil analgésicodotramadol através da verificação temporal de sua concentração plamática em cadelas submetidas à ovário-salpingohisterectomia. São Paulo, 2006. 88f. Dissertação. (Mestrado em Ciências) - Faculdade de Medicina Veterinária e Zootecnia. Universidade de São Paulo, São Paulo, 2006.

FIRTH AM, HALDANE SL. Development of a scale to evaluate postoperative pain in dogs. Journal of the American Veterinary Medical Association,v.214, n. 5, p .651-659, 1999.

HANSEN, B. D. Assessment of pain in dogs: veterinary clinical studies. ILAR Journal, v. 44, n.3, p.197-205, 2003.

HELLYER, P. W. Pain identification. In: ETTINGER, S. J.; FELDMAN, C. E. Textbook of Veterinary Internal Medicine. 6. ed. Philadelphia: Elsevier Saunders, 2005. p. 16-21.

HELLYER, P.W.; ROBERTSON, S.A.; FAILS, A.D. Pain and its management. In: Tranquilli, W. J., Thurmon, J. C., \& Grimm, K. A. Veterinary Anesthesia and analgesia. 4 ed. 2007. Iowa: Blackwell. Cap. 3. P. 31-60.

IMAGAWA, V.H. Avaliação da eficácia analgésica de três doses diferentes da dipirona sódica em cadelas submetidas à ovariosalpingohisterectomia. São Paulo, 2006. 122f. Tese. (Doutorado em Ciências) - Faculdade de Medicina Veterinária e Zootecnia. Universidade de São Paulo, São Paulo, 2006.

LASCELLES BDX, BUTTERWORTH SJ, WATERMAN AE. Postoperative analgesic and sedative effects of carprofen and pethidine in dogs.Veterinary Record, v. 134, n.7, p. 187-91, 1994. 
LUNA, S.P.L. Dor, senciência e bem-estar em animais: sensiência e dor. Ciência Veterinária nos Trópicos, v.11, p. 17-21, 2008. Suplemento 1.

MATHEWS K. A. Management ofpain. Veterinary Clinics of North America: Small Animal Practice. $2000 ; 30(4): 729-55$.

MICH, P.M.; HELLYERP.W. Métodos objetivos e categóricos para avaliar a dor e analgesia. . In: GAYNOR, J.S.; MUIR III, W.W. Manual de controle da dor em Medicina Veterinária. São Paulo, Roca. 2009. Ed MedVet. Cap. 6. P. 78-112.

MORTON, C.M.; REID, J.; SCOTT, E.M.; HOLTON, L.L.;NOLAN, A.M. Application of a scaling model to establish and validate an interval level pain scale for assessment of acute pain in dogs. American JournalofVeterinaryResearch, v.66, n. 12, p. 2154-2166, 2005.

MURREL, J.C.; PSATHA, E.M.; SCOTT, J. et al. Application of a modified form of the Glasgow pain scale in a veterinary teaching centre in the Netherlands. Veterinary Record, v.29, p.403-408, 2008. 